\title{
LONG TERM IMPROVEMENTS IN CHEMOTHERAPY INDUCED PERIPHERAL NEUROPATHY- OBJECTIVE IMPROVEMENT OR PATIENT ADAPTATION?
}

Tiffany Li; Hannah C Timmins; Michelle Harrison; Lisa G Horvath; Tracy King; Peter Grimison; David Goldstein; THE UNIVERSITY OF SYDNEY Matthew C Kiernan; Susanna B Park.

\section{Introduction}

- Chemotherapy-induced peripheral neuropathy is a disabling side effect of neurotoxic chemotherapy that persists and affects cancer survivors' quality of life.

- Patient reported outcomes (PROs) are increasingly recognised as important in evaluating the impact of neuropathy.

- However, questions remain on whether patient reported improvements in the long-term reflect objective improvements or patient adaptation.

\section{Methods}

- Forty-two patients (10 males; mean age $=54.4 \pm 11.9$ years) were assessed prospectively prior to neurotoxic chemotherapy treatment (taxanes, platinums, or bortezomib), at 3-months post completion (mean $=3.0$ \pm 0.8 months) and at 9 -months post completion (mean $=9.5 \pm 2.9$ months) of treatment.
Royal Prince Alfred Hospital
UNSWW

\section{Assessment Tools}

- The FACT/GOG NTX is a validated PRO used to assess neurological complications in the cancer patient cohort.

- The grating orientation task (GOT) and 2point discrimination task (2PD) are objective tasks assessing sensory function in the upper and lower limbs respectively (Figure 1).
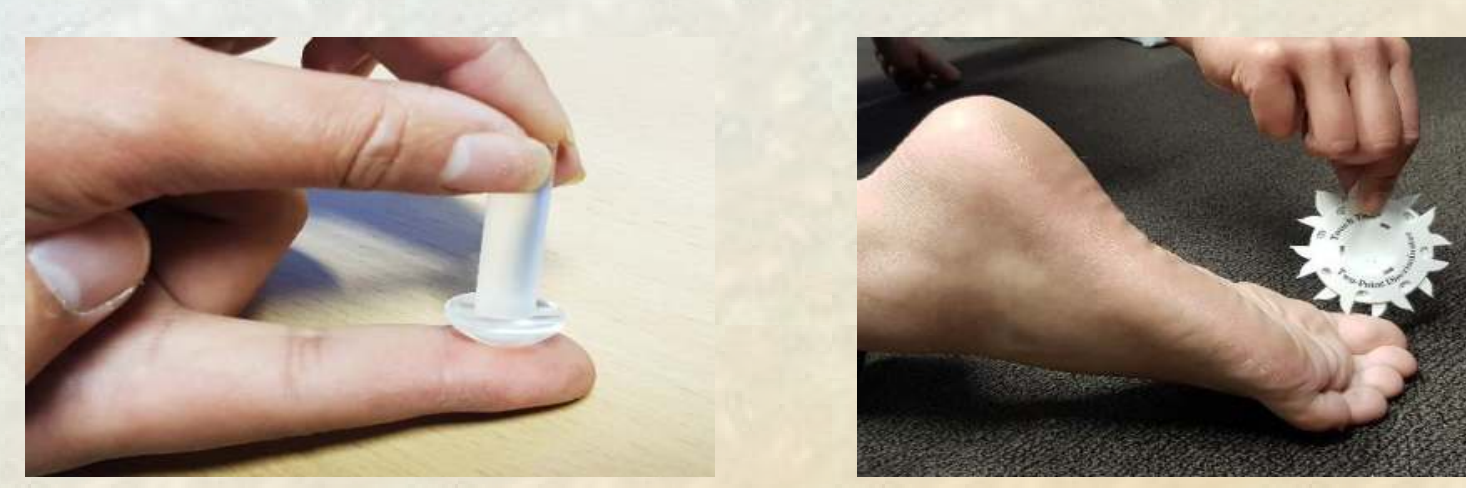

Figure 1. (Left to right) Grating orientation task and 2-point discrimination task

\section{Results}

- At 3-months post completion, patients reported significantly more numbness and tingling in the hands $(p<0.01)$ and feet $(p<0.01)$ than at baseline (Figure $2 A)$.

- These results are reflected in objective tasks, demonstrated by significant decline in GOT $(p=0.01)$ and $2 \mathrm{PD}(\mathrm{p}<0.01)$ performance with elevated sensory thresholds 3 -months post completion (Figure 2B,C).

- At 9-months post completion, patients reported significant symptomatic improvement with less numbness and tingling in the hands $(p=0.02)$ and feet $(p<0.01)$ than at 3 -months follow-up (Figure $2 A)$.

- However objective tasks demonstrated no significant improvements (GOT $p=0.20,2 P D p=0.29$, Figure $2 B, C)$.

A

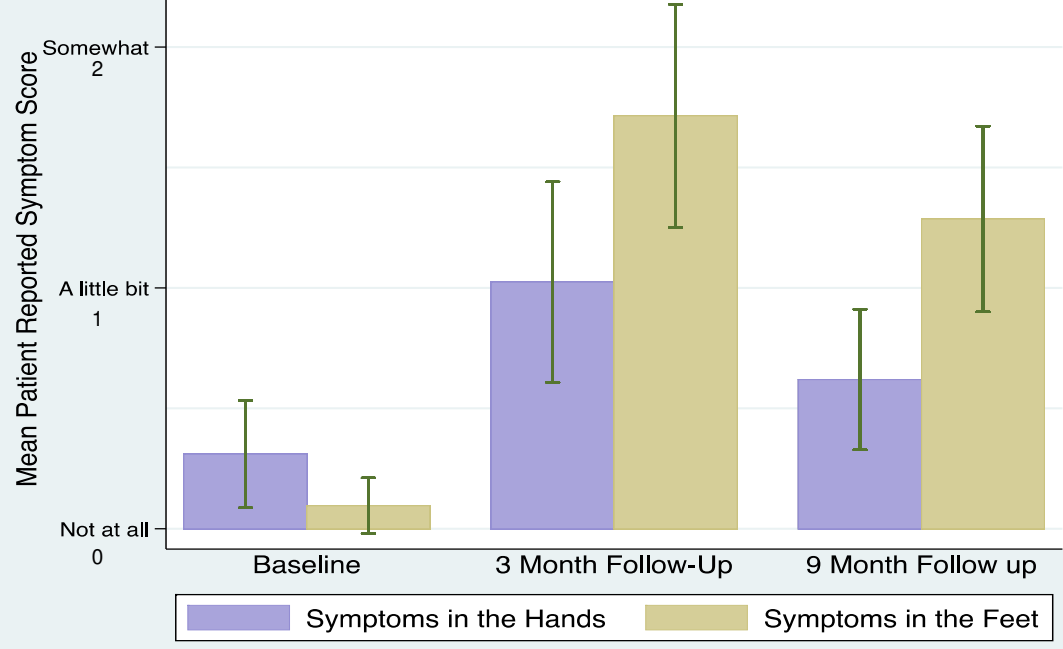

Figure 2. (Left to right) Mean score ( \pm SEM) of neuropathy symptoms on the FACT-GOG NTX (question 1 and 2), mean GOT threshold score ( \pm SEM), mean 2PD score ( \pm SEM) at 3 time points
C

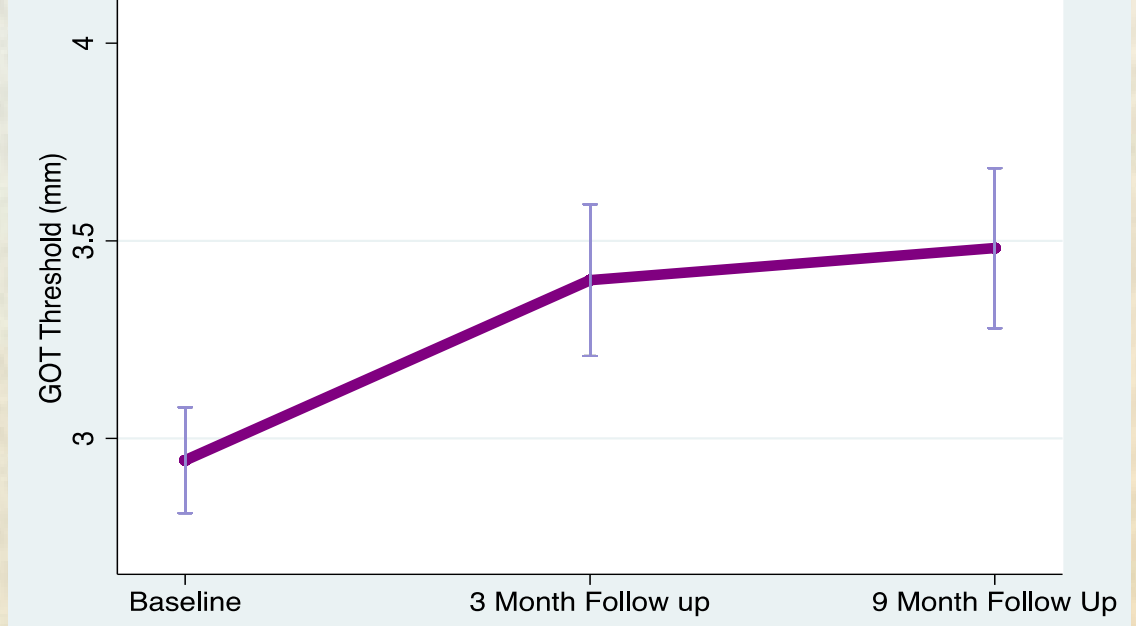

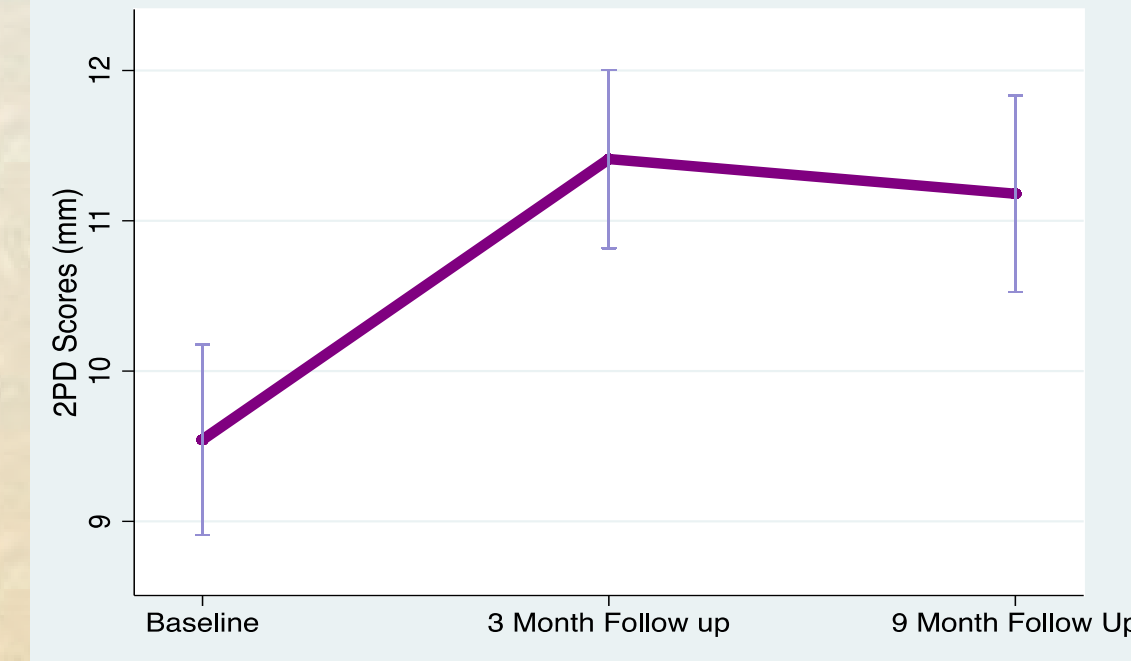

\section{Conclusion}

- Patient reported improvements may result from symptom adaptation rather than objective physiological improvements.

- Education and intervention to support adaptation is vital to ensure patients with neuropathy are able maintain high quality of life.

- PROs should be used alongside objective measures to both capture patient experience and to quantify neurological deficits. 